$\mathcal{O}_{\text {doi: } 10.3765 / \mathrm{sp} .3 .2}^{\text {Semantics \& Pragmatics Volume 3, Article 2: 1-13, } 2010}$

\title{
Embedded Implicatures and Experimental Constraints: A Reply to Geurts \& Pouscoulous and Chemla*
}

\author{
Uli Sauerland \\ Zentrum für Allgemeine \\ Sprachwissenschaft, Berlin
}

Received 2009-11-13 / First Decision 2009-11-22 / Revised 2009-12-11 / Accepted 2009-12-08 / Published 2010-01-25

\begin{abstract}
Experimental evidence on embedded implicatures by Chemla (2009b) and Geurts \& Pouscoulous (2009a) has fewer theoretical consequences than assumed: On the one hand, the evidence successfully argues against obligatory local implicature computation, which has however already been discredited. On the other hand, the data are fully consistent with optional local implicature computation.
\end{abstract}

Keywords: conversational implicature, embedded implicature, experimental pragmatics, free-choice permission, truth dominance

Both Chemla (2009b) (C in the following) and Geurts \& Pouscoulous (2009a) (G\&P in the following) in recent papers in this journal provide welcome new experimental evidence on embedded implicatures. However, while their work takes us a couple of steps closer to full understanding of the issue, I will argue that in both papers the theoretical implications of the new data are overstated and much work remains to be done.

Intuitively clear cases of embedded implicatures are examples like (1).

(1) a. If you ate some of the cookies and no one else at any, then there must still be some left. (Levinson 2000: 205)

b. Mary solved the first problem or the second problem or both problems.

(Chierchia et al. 2008: (31))

* I thank Nicole Gotzner, Lisa Hartmann and the editors of this journal for their help with this paper, and the German Research Foundation (DFG grant SA 925/1 in the Emmy Noether Programm) for financial support.

(C)2010 Uli Sauerland

This is an open-access article distributed under the terms of a Creative Commons NonCommercial License (creativecommons.org/licenses/by-nc/3.o). 
Here the implicatures of some and or are part of the truth-conditional content of an embedded sentence: the conditional in (1a), which is understood as if you ate some and not all of the cookies, and a disjunct in (1b), which is understood as Mary solved either the first problem or the second problem and not both. In these examples, the sentence without the embedded implicature would be either contradictory (If you ate some or all of the cookies, then there must still be some left) or a violation of a pragmatic constraint (\#Mary solved at least one of the problems or both problems, see Singh 2008).

The question theorists of all stripes are faced with is whether and how to integrate these phenomena into a general theory of implicatures, or at least of quantity or scalar implicatures. Some narrower directions that have been pursued to address the general question raised by embedded implicatures are listed in (2).

(2) I. How frequently and under what conditions do embedded implicatures arise?

II. Are embedded implicatures a uniform phenomenon? Or more specifically: How many mechanisms can give rise to embedded implicatures?

III. Are embedded implicatures really implicatures? Or more specifically: When is the mechanism giving rise to embedded implicatures the same one as the mechanism giving rise to global implicatures?

G\&P and C both focus on the first of these directions, and take this discussion as far as it can be taken presently. However, I argue that just pursuing the first direction is insufficient to resolve the issues embedded implicatures raise fully. In particular, I show that independent pragmatic constraints - in particular, the constraint of Truth Dominance (Meyer \& Sauerland 2009) - predict conditions on when embedded implicatures can be detected that are largely independent of the account of embedded implicatures assumed. Therefore, the observations on the presence and absence of (embedded) implicatures by G\&P and C are consistent with much wider range of theories of implicatures than what the original papers say. In the second section of the paper, I address a finding by $\mathrm{C}$ on the embedding of free-choice effects that speaks to directions II and III of (2). I argue that this finding is more significant for the account of embedded implicatures and speculate on two theoretical ideas that would account for it. I conclude that C's second result is the most important one for the theory of implicatures from these two papers. 
Embedded Implicatures and Experimental Constraints

\section{Embedded Implicatures are Still Repairs}

G\&P are exclusively concerned with the first issue of (2). The primary target of G\&P is an extreme view of localism espoused by Levinson (2000) and Chierchia (2004). ${ }^{1}$ The Levinson/Chierchia view predicts that implicatures should always be fully local unless a cancellation mechanism applies. A number of people have noted that this prediction seems to be intuitively wrong in many cases. Specifically, this holds in case the embedded implicature is not needed to make the sentence coherent (see for instance Geurts 2009; Russell 2006; Sauerland 2004b). Compare (3) with (1): Intuitively, (3a) does not seem to mean the same as If you ate some but not all of the cookies, then you must have liked them. And for the multiple disjunction in (3b), the paraphrase Mary solved either exactly one or all three of the problems, which local computation of implicatures predicts, is clearly off the mark.

(3) a. If you ate some of the cookies, then you must have liked them.

b. Mary solved the first problem or the second problem or the third problem.

In these two examples, the addition of a local implicature to the truth conditions results in weaker truth conditions for the entire sentences - in (3a) the implicature trigger occurs in a downward entailing environment, but not in (3b). Such examples show that local implicatures cannot be obligatory. ${ }^{2}$

Examples where local implicatures would cause truth conditions that are stronger overall are the focus of G\&P. The data presented by G\&P shows to my full satisfaction that the prediction of the proposals of Levinson and Chierchia is wrong also for these cases. Their result is also consistent with other experimental results presented by Chemla (2009a), Schwarz, Clifton \& Frazier (2008) and Bezuidenhout, Morris \& Widman (2009), who look at different environments: mostly downward entailing cases like negative attitude verbs, the restrictor of a universal quantifier and conditional, but Bezuidenhout et al. (p. 139) also look at the scope of conditionals and present some findings similar to G\&P, though less striking. In sum, the proposals of

1 I am not sure whether any researcher active in this area still holds this extreme view: The unpublished paper by Chierchia et al. (2008) that G\&P cite seems not fully consistent to me in this regard (Sauerland submitted): initially it adopts the view of Chierchia (2004), however, later the quite different view of Fox (2007) is assumed without any comment on the shift.

2 Acknowledging this problem, Chierchia (2004) proposes that cancellation of implicatures is obligatory in downward entailing environments. Still, (3b) remains a problem for Chierchia's proposal. 
Levinson (2000) and Chierchia (2004) are falsified by the experimental data to the extent possible. ${ }^{3}$

G\&P claim their results also argue against another view, which they call Minimal Conventionalism. However, I will show that G\&P are mistaken: Actually, their result says nothing about Minimal Conventionalism once we take into account general pragmatic constraints on how ambiguous sentences are judged. To show this, I consider the view of Fox (2007) as a concrete example of G\&P's Minimal Conventionalism. I motivate the general pragmatic principle of Truth Dominance and then argue that G\&P's data are fully consistent with Fox's (2007) account once Truth Dominance is taken into account.

Fox's (2007) account is non-committal on the locality of implicature computation, allowing it to apply locally, but also globally. He assumes that implicatures can be contributed to the meaning of a sentence by the grammatical operator Exh. ${ }^{4}$ (Fox 2007: p. 79 \& p. 97) defines the Exh operator via the three statements in (4) through (6) (with minor notational adjustments). The operator depends on a contextually provided set of alternative propositions $C$, which can be taken to be the scalar alternatives of the argument of Exh in the examples in the following.

(4) $\quad \mathrm{NW}_{C}(p)=\{q \in C \mid p$ does not entail $q\}$

(5) $\quad q$ is innocently excludable given $C$ if and only if

$$
\neg \exists q^{\prime} \in \mathrm{NW}_{C}(p)\left[[p \wedge \neg q] \rightarrow q^{\prime}\right]
$$

(6) $\quad \llbracket \mathbf{E x h} \rrbracket_{C}(p)(w) \Leftrightarrow p(w)$

$\& \forall q \in \mathrm{NW}_{C}(p)[[q$ is innocently excludable given $C] \rightarrow \neg q(w)]$

Consider now Fox's (2007) account for example (7). The account predicts an ambiguity between a local+global reading, which corresponds to structure (8a), and a global-only reading, which corresponds to structure (8b).

(7) All the squares are connected with some of the circles. (G\&P: (26a))

3 Of course, there are always ways to save any scientific theory by adding additional assumptions, but nothing short of almost obligatory local cancellation of the proposed obligatory local implicatures would seem to do the trick in this case.

4 There are two major differences between Fox's account and that of Chierchia (2004): First, Fox does not require local application of implicature computation. And second, his Exh operator is different from Chierchia's due to the appeal to innocent excludability. The second difference does not matter for the following, but is important for the analysis of disjunctions (Sauerland submitted). 
Embedded Implicatures and Experimental Constraints

(8) a. Exh All the squares $\lambda x \mathbf{E x h} x$ be connected with some of the circles.

b. Exh All the squares $\lambda x x$ be connected with some of the circles.

The two readings stand in a special logical relationship: the local-global reading logically entails the weaker, global-only reading. From work on scope ambiguity resolution, it is independently known that speakers' intuitions are affected by the entailment relation between the two readings. I adopt the principle Truth Dominance from Meyer \& Sauerland (2009) to account for this effect because one case they consider is exactly analogous to (8), ${ }^{5}$ namely, the German example (9). Most theories of quantifier scope in German predict (9) to be ambiguous between two structural representations that should give rise to the two readings given below (9). However, previous researchers (Büring \& Hartmann 2001; Reis 2005) have noted that (9) seems to lack the second one of these readings: the reading where the postverbal subject takes scope over the sentence-initial object (the every $\gg$ only reading in (9)).

(9) Nur Maria liebt jeder.

only Mary[ACC] loves everyone.NOM

only $\gg$ every: $\forall y(y=\operatorname{Mary} \leftrightarrow \forall x \operatorname{love}(x, y))$

[every $\gg$ only: $\forall x \forall y(y=\operatorname{Mary} \leftrightarrow \operatorname{love}(x, y))$ ]

Meyer \& Sauerland (2009) explain the lack of evidence for the every $\gg$ only reading by arguing that this reading cannot be detected for pragmatic reasons. Specifically, the Truth Dominance principle in (10) predicts it to be undetectable: Because the strong, only $\gg$ every reading entails the weak, every $\gg$ only reading, any situation where the truth values of the two readings differ is one where the strong reading is false while the weak one is true. But Truth Dominance predicts that in such a situation, speakers will judge the sentence to be true, as it's predicted to be by the weak reading. The strong reading therefore remains undetectable in the truth conditions of (9).

(10) Truth Dominance: Whenever an ambiguous sentence $\mathrm{S}$ is true in a situation on its most accessible reading, we must judge sentence $S$ to be true in that situation.

(Meyer \& Sauerland 2009: (1))

5 The principle can be traced back at least to work on wide scope indefinites by Abusch (1994). Gualmini, Hulsey, Hacquard \& Fox (2008) call a similar principle Charity. The differences between the Charity and Truth Dominance are not relevant to the discussion in this paper. In fact, Charity would make exactly the same predictions as Truth Dominance for the examples in the following. 
Principle (10) is a well-supported pragmatic principle: As already mentioned, work by Abusch (1994) and Gualmini et al. (2008) provides further support for a principle like (10) and in addition, principle (10) makes pragmatic sense as a principle of cooperative behavior in discourse.

Principle (10) is directly relevant for determining the predictions of Fox's (2007) analysis of implicatures in the following way. As discussed above, Fox's account predicts (7) to be ambiguous between the two readings represented in (8). However, reading (8a) entails (8b), so the same situation obtains as with the two readings of (9). Principle (10) entails for (7) that only reading (8b) can be empirically detected for $(7){ }^{6}$

Indeed, G\&P argue that only reading (8b) is empirically supported by the judgments of the subjects in their experiments, which is the judgment that Fox's ambiguity account together with Truth Dominance predict. The experimental results of G\&P are therefore fully consistent with Fox's proposal and what G\&P call Minimal Conventionalism more generally.

The preceding discussion does not entail that Fox's (2007) account is without problems: Fox's account makes the wrong prediction for cases like (3) because the local application of implicature computation leads to a weaker interpretation than the one actually attested. In this case, the local implicature would be detectable, but is actually not attested. Fox briefly entertains two suggestions that would address this shortcoming (Fox 2007: page 82), but both fall short. One suggestion is to only compute local implicatures if they strengthen the sentence meaning. The other suggestion is to only permit global application of his Exh operator. Both of these suggestions solve the problem of (3), but leave Fox with no account for (1). So, Fox's (2007) account would need to be amended further. For example, the empirical problems would be solved by stipulating that embedded Exh is blocked unless an inconsistency or pragmatic violation results otherwise. ${ }^{7}$ The important point for our present purposes, though, is that G\&P's data do not bear on Fox's (2007) account and probably others that G\&P would characterize as Minimal Conventionalism. The above discussion also shows how difficult it is to address the puzzle posed by embedded implicatures by just looking at the distribution of embedded implicatures. I conclude

6 To be more precise, the application of Truth Dominance here assumes that (8b) represents a more accessible syntactic parse than (8a). Since (8b) contains fewer silent operators, this assumption is independently justified.

7 This is essentially a more specific statement of the view of Sauerland (2004a) that embedded implicatures are a repair strategy. 
Embedded Implicatures and Experimental Constraints

therefore that a comparison of the properties of embedded implicatures with non-embedded ones may be a more promising direction to pursue than to solely focus on the distribution of embedded implicatures. Such attention to the properties of implicatures would address both II and III of the questions in (2). In the next section, I focus on one aspect of the data reported by $\mathrm{C}$ that points in this direction.

\section{Are Embedded Implicatures Implicatures?}

The results of C (= Chemla 2009b) add one new aspect, but are otherwise consistent with the picture already summarized: The results show that obligatory localism is false, but don't distinguish between other views. In particular, C's discussion of examples like (11a) and (11b) is limited in the same way as the discussion of (9) by G\&P: the only theory Chemla's result argues against is the extreme localism of Levinson (2000) and Chierchia (2004), which is already known to have numerous problems. More viable views of localism, where embedded implicatures are an option, but not required, make exactly the right predictions for both examples in (11) - namely, the same predictions as a global account.

(11) a. Every student read some of the books.

b. No student read all the books.

The most interesting result of C's study is the embedded free choice effect in examples like (12). He shows experimentally that subjects judge (12) to entail that every student is allowed to have an apple and also that every student is allowed to have a banana.

(12) Every student is allowed to have an apple or a banana. (C: (12b))

Chemla's observation is interesting because it shows a difference between free choice effects and scalar implicatures, which are not as frequently locally computed in the same environment. This difference may bear on the second and third of the questions in (2). Unfortunately, Chemla's theoretical discussion is limited to the account of Fox (2007) and on one point even mistaken. Chemla compares the two versions of Fox's (2007) proposal I already mentioned above: either permitting embedded occurrences of Exh or restricting Exh to one occurrence with clausal scope per utterance. The non-deterministic former view predicts (12) to be ambiguous between the two 
Uli Sauerland

representations in (13), while the latter globalist view permits only representation (13b)

(13) a. Exh Every student $\lambda x x$ is allowed to have an apple or a banana.

b. Exh Every student $\lambda x \mathbf{E x h} x$ is allowed to have an apple or a banana.

Chemla focuses on the fact that the Fox's globalist view incorrectly predicts that (12) should be restricted to scenarios where not all students make the same choice since (13a) entails that neither every student is allowed to have an apple nor every student is allowed to have a banana. What Chemla fails to note, though, is that the optionally local version of Fox's proposal also predicts (13a) as a possible reading for (12). In particular, neither does (13a) entail (13b), nor vice-versa, and therefore both readings should be detectable. But this doesn't seem to be the case and therefore (12) is also a problem for the non-deterministic version of Fox's proposal, not just for the global one. Since I argued above that both version are independently problematic, Chemla's new evidence just strengthens the point against both proposals.

The main conclusions I draw from Chemla's paper concern a) the status of free choice inferences, and b) the relation of embedded to global implicatures. Chemla's data only speak to my questions in (2) if we assume that free choice inferences are indeed implicatures. Chemla's data actually cast this relationship in doubt. There is not that much empirical evidence in favor of the relationship in the first place: the main direct piece of evidence in favor of an implicature account of free choice inferences is the observation by Kratzer \& Shimoyama (2002) that the inferences disappear in the scope of negation just like implicatures. ${ }^{8}$ However, C shows two differences between free choice inferences and implicatures: First, only free choice inferences are locally present in the scope of a universal quantifier as I already referenced above. Second, C shows that negated modalized statements like (14a) don't trigger free choice inferences. Since (14b) is logically equivalent to (14a) the absence of a free choice inference in (14b) shows that free-choice inferences are not detachable in the sense of (Grice 1989). Usually implicatures are detachable as, for example, Grice already discusses.

(14) a. John is allowed to not do A or not do B.

b. John is not required to do A and B. (Chemla 2009b: (15a))

8 Furthermore, free choice inferences can also be cancelled like other implicatures as in You may have an apple or a banana, but I don't know which. 
Embedded Implicatures and Experimental Constraints

C's results are intuitively plausible and very interesting for the theory of free-choice inferences. As far as I can see, there are two possible directions to pursue. On the one hand, one could seek to treat free choice inferences not as implicatures. Specifically, C's result could be seen to support non-implicature accounts of free choice such as Zimmermann (2000). On the other hand, it may be that matrix free-choice effects are still implicatures, but embedded free choice effects may be due to a special free-choice inference generating operator. The latter position should be attractive to those who believe that there are satisfying analysis of free-choice inferences as an implicature (Fox 2007; Schulz 2005).

\section{Conclusions}

In sum, the recent experimental work by G\&P and $C$ has confirmed the views of those who have argued against the obligatory localism of Levinson (2000) and Chierchia (2004), e.g. Geurts (2009), Russell (2006), and Sauerland (2004b). Beyond that, the account of embedded implicatures and their relation to global implicatures are still unclear. Solely testing for the presence of embedded implicatures as G\&P and C mostly do may be insufficient for understanding embedded implicatures. Rather it may be more promising to investigate wether the content of embedded implicatures is exactly the same as that of implicatures at the matrix level.

In this direction, the difference $\mathrm{C}$ observes between embedded and matrix implicatures is interesting and most likely helpful in sorting out the puzzle of embedded implicatures. While I have no complete account to offer myself, I close with some arguments to be skeptical of Geurts \& Pouscoulous (2009b) analysis of Chemla's example (12): Geurts \& Pouscoulous (2009b) suggest accounting for (12) as an instance of an embedded speech act. This account, I argue now is plausible for some cases, but most likely cannot cover all cases of embedded implicatures: The possibility of embedded speech acts has been acknowledged at least since Huddleston (1973) and embedded speech acts can certainly be a source of embedded implicatures: ${ }^{9}$ (15) illustrates that embedded speech acts must trigger embedded implicatures: the modal particle wohl ('well') requires an embedded speech act interpretation for the complement of glaubt ('believes') and furthermore triggers an inference that

9 The idea of a metalinguistic negation of Horn (1985) is closely related to the idea of embedded implicatures, but more specific since it assumes a restriction to negation. 
Uli Sauerland

the speaker also believes the complement clause.

(15) \#Bill glaubt, dass einige der Kinder wohl krank sind. Aber alle Bill believes that some of the children wohl sick are but all Kinder sind krank. children are sick.

However, this alone doesn't predict correctly that (15) is odd. The oddness of (15) is only predicted if there is also an embedded implicature. The embedded implicature is the reason that the stronger belief, that some, but not all children are sick, is attributed to the speaker. Then (15) is predicted to be odd because the second sentence explicitly contradicts this attribution of the embedded implicature to the speaker.

This example indicates that embedded speech acts trigger embedded implicatures as all theories of speech acts would predict. ${ }^{10}$ However, I do not believe that the reverse entailment also holds - that an embedded implicature is always triggered by an embedded speech act. One problem for this entailment is the following: Krifka (2001) argues that most does not allow embedding of speech acts in its scope. Hence, (16) should not allow embedded free choice inferences unlike (12). However, this doesn't accord with my intuitions: (16) suggests that most students can choose freely. For instance, consider (16) in the following scenario: the majority of students can freely choose between A and B and the few other students, who cannot freely choose, must do option A. In this scenario (16) seems acceptable to me, even though it may happen that not a single student chooses option B. The acceptability of (15) in such a scenario is only expected if the free choice inference is embedded in the scope of most.

Most students are allowed to do A or B.

The embedded free choice inferences in (16) couldn't be due to an embedded speech act if Krifka's (2001) is correct that most blocks embedded speech acts. Therefore, (16) presents a problem for the proposal to derive all embedded implicatures from embedding of speech acts. Some further data that are problematic for the idea of deriving all embedded implicatures from embedded speech acts are discussed by Sauerland (2004a). Therefore, I conclude that contrary to Geurts \& Pouscoulous's (2009b) opinion, Chemla's

10 This prediction, of course, arises to the extent that theories of speech acts permit embedding of speech acts in the first place. 
Embedded Implicatures and Experimental Constraints

data in (12) are still in need of an account. And the search for such an account may finally really lead us to a better understanding of embedded implicatures.

\section{References}

Abusch, Dorit. 1994. The scope of indefinites. Natural Language Semantics 2(2). 83-135. doi:10.1007/BFo1250400.

Bezuidenhout, Anne, Robin Morris \& Cintia Widman. 2009. The DE-blocking hypothesis: The role of grammar in scalar reasoning. In Sauerland \& Yatsushiro (2009), 124-144.

Büring, Daniel \& Katharina Hartmann. 2001. The syntax and semantics of focus-sensitive particles in German. Natural Language \& Linguistic Theory 19(2). 229-281. doi:10.1023/A:1010653115493.

Chemla, Emmanuel. 2009a. An experimental approach to adverbial modification. In Sauerland \& Yatsushiro (2009), 249-263.

Chemla, Emmanuel. 2009b. Universal implicatures and free choice effects: Experimental data. Semantics and Pragmatics 2(2). 1-33. doi:10.3765/sp.2.2. Chierchia, Gennaro. 2004. Scalar implicatures, polarity phenomena, and the syntax/pragmatics interface. In Adriana Belletti (ed.), Structures and beyond, 39-103. Oxford, UK: Oxford University Press.

Chierchia, Gennaro, Benjamin Spector \& Danny Fox. 2008. The grammatical view of scalar implicatures and the relationship between semantics and pragmatics. Ms, to appear in Maienborn, Claudia, Klaus von Heusinger, and Paul Portner (eds.) Handbook of Semantics.

Fox, Danny. 2007. Too many alternatives: Density, symmetry and other predicaments. Semantics and Linguistic Theory 17. doi:1813/11295.

Geurts, Bart. 2009. Scalar implicature and local pragmatics. Mind and Language 24(1). 51-79. doi:10.1111/j.1468-0017.2008.01353.x.

Geurts, Bart \& Nausicaa Pouscoulous. 2009a. Embedded implicatures?!? Semantics and Pragmatics 2(4). 1-34. doi:10.3765/sp.2.4.

Geurts, Bart \& Nausicaa Pouscoulous. 2009b. Free choice for all: a response to Emmanuel Chemla. Semantics and Pragmatics 2(5). 1-10. doi:10.3765/sp.2.5.

Grice, Paul. 1989. Studies in the way of words. Cambridge, MA: Harvard University Press.

Gualmini, Andrea, Sarah Hulsey, Valentine Hacquard \& Danny Fox. 2008. The 
question-answer requirement for scope assignment. Natural Language Semantics 16(3). 205-237. doi:10.1007/s11050-008-9029-z.

Horn, Laurence R. 1985. Metalinguistic negation and pragmatic ambiguity. Language 61(1). 121-174.

Huddleston, Rodney. 1973. Embedded performatives. Linguistic Inquiry 4(4). 539-541.

Kratzer, Angelika \& Junko Shimoyama. 2002. Indeterminate pronouns: The view from Japanese. In Yukio Otsu (ed.), Proceedings of the Third Tokyo Conference on Psycholinguistics, 1-25. Tokyo: Hituzi Syobo.

Krifka, Manfred. 2001. Quantifying into question acts. Natural Language Semantics 9(1). 1-40. doi:10.1023/A:1017903702063.

Levinson, Stephen C. 2000. Presumptive meanings. Cambridge, Mass.: MIT Press.

Meyer, Marie-Christine \& Uli Sauerland. 2009. A pragmatic constraint on ambiguity detection: A rejoinder to Büring and Hartmann and to Reis. Natural Language \& Linguistic Theory 27(1). 139-150. doi:10.1007/s110490o8-9060-2.

Reis, Marga. 2005. On the syntax of so-called focus particles in German: a reply to Büring and Hartmann 2001. Natural Language \& Linguistic Theory 23(2). 459-483. doi:10.1007/s11049-004-0766-5.

Russell, Ben. 2006. Against grammatical computation of scalar implicatures. Journal of Semantics 23(4). 361-382. doi:10.1093/jos/ffloo8.

Sauerland, Uli. 2004a. On embedded implicatures. Journal of Cognitive Science 5(1). 107-137.

Sauerland, Uli. 2004b. Scalar implicatures in complex sentences. Linguistics and Philosophy 27(3). 367-391. doi:10.1023/B:LING.0oooo23378.71748.db.

Sauerland, Uli. submitted. Disjunction and implicatures: Some notes on recent developments. In Chungmin Lee (ed.), Proceedings of the CIL18 workshop on contrastiveness in information structure and/or scalar implicatures.

Sauerland, Uli \& Kazuko Yatsushiro (eds.). 2009. Semantics and pragmatics: From experiment to theory. Basingstoke, UK: Palgrave Macmillan.

Schulz, Katrin. 2005. A pragmatic solution for the paradox of free choice permission. Synthese 147(2). 343-377. doi:10.1007/s11229-005-1353-y.

Schwarz, Florian, Charles Jr. Clifton \& Lyn Frazier. 2008. Strengthening 'or': Effects of focus and downward entailing contexts on scalar implicatures. To appear in UMOP 37.

Singh, Raj. 2008. On the interpretation of disjunction: Asymmetric, incremental, and eager for inconsistency. Linguistics and Philosophy 31(2). 
Embedded Implicatures and Experimental Constraints

245-26o. doi:10.1007/s10988-008-9038-x.

Zimmermann, Thomas Ede. 2000. Free choice disjunction and epistemic possibility. Natural Language Semantics 8(4). 255-290. doi:10.1023/A:1011255819284.

Uli Sauerland

Zentrum für Allgemeine Sprachwissenschaft

Schützenstr. 18

D-10117 Berlin

uli@alum.mit.edu 